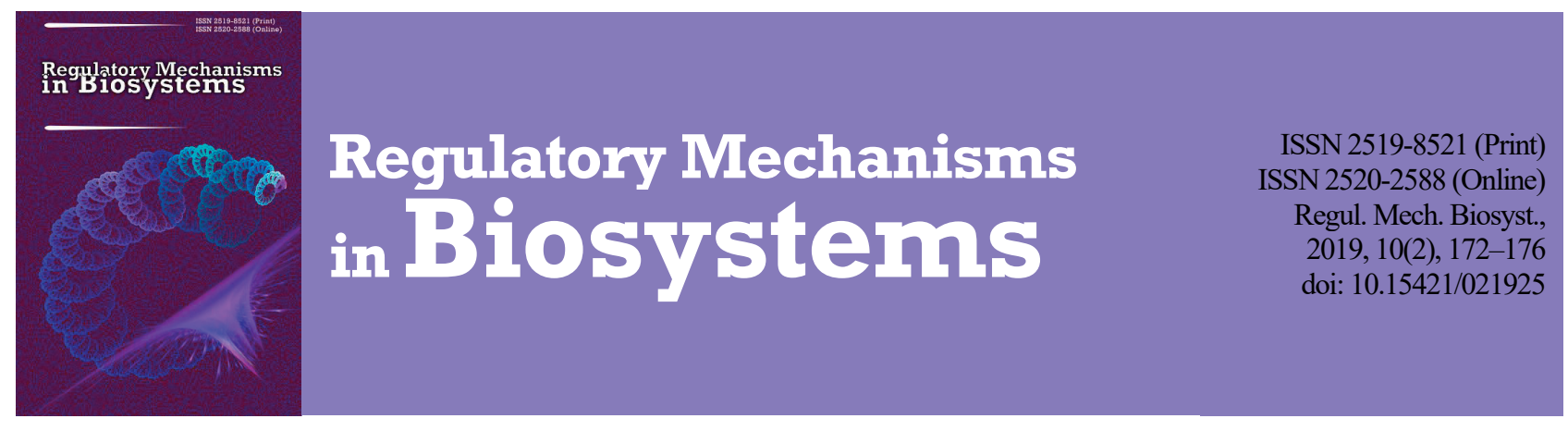

\title{
Vaginal candidiasis among women of reproductive age in Dnipro city (Ukraine): Species spectrum of Candida and their susceptibility to antimycotics
}

\author{
O. M. Starishko, Y. S. Voronkova, T. H. Ostanina, O. S. Voronkova
}

Oles Honchar Dnipro National University, Dnipro, Ukraine

Article info

Received 11.03.2019

Received in revised form 24.04.2019

Accepted 26.04.2019

Oles Honchar Dnipro National University,

Gagarin av., 72,

Dnipro, 49010, Ukraine.

Tel.: + 38-096-225-04-63.

E-mail:voronkova_olga@i.ua

\begin{abstract}
Starishko, O. M., Voronkova, Y. S., Ostanina, T. H., \& Voronkova, O. S. (2019). Vaginal candidiasis among women of reproductive age in Dnipro city (Ukraine): Species spectrum of Candida and their susceptibility to antimycotics. Regulatory Mechanisms in Biosystems, 10(2), 172-176. doi:10.15421/021925

Candidiasis is one of the most common pathological processes, especially among women of reproductive age, especially pregnant. It can be expressed as Candida carriage and as an active form of infection. Although candidiasis is caused by opportunistic microorganisms, its effects can be quite significant, so it is necessary to treat it with the use of antimycotic drugs. In view of the spread among these microorganisms of resistance to antimicrobial drugs, the determining factor for the success of therapeutic measures, it is important to make a preliminary assessment of the susceptibility to antimycotics of strains isolated from persons with Candida carriage or active candidiasis. The aim of the research was to study the species range of fungi of the genus Candida isolated from the reproductive tract in women with dysbiosis and to study their susceptibility to antimycotics. Total of 227 samples of biological material from women aged 16 to 56 years have been investigated. Microscopic and cultural methods of detecting of fungi in the primary material were used for research. For the identification of species of Candida fungi, a polymerase chain reaction method in real-time was used. It was determined that the frequency of detection of Candida fungi in the composition of vaginal microbiome during inflammatory pathological processes was $76.7 \%$. It was shown that the incidence of Candida carriage $51.7 \%$ - slightly exceeded the frequency of detection of the active form of infection $-48.3 \%$. The species spectrum of pathogens included: C. albicans (57.1\%), C. krusei (12.6\%), C. glabrata (19.5\%) and Candida spp. (16.1\%). In the case of active form of infection, C. albicans was prevalent $-64(76.2 \%)$ cases. In the case of Candida carriage, other species were prevalent -58 (64.4\%) cases. The most effective drug against all isolates was amphotericin B - more than $87 \%$ of isolates were susceptible. The least effective drug was nystatin: less than $60 \%$ of isolates were susceptible. There is a rather high level of resistance to certain antimycotic drugs among the Candida species, which requires a prior preliminary study of the susceptibility of isolated strains to antimycotics for the purpose of choosing a rational and effective treatment scheme.
\end{abstract}

Keywords: Candida; candidiasis; carriage; species spectrum; incidence rates; antimycotic susceptibility.

\section{Introduction}

Vulvovaginal candidiasis is one of the most common pathological processes in the structure of inflammatory diseases of female genital organs. It is known that among the infections of the lower reproductive system up to $50 \%$ of cases of lesions are caused by Candida (Richter et al., 2005; Rathod et al., 2012). As described by Rathod \& Buffler (2014), $75 \%$ of women will experience an episode of vulvovaginal candidiasis in their lifetimes, $50 \%$ of whom will experience at least a second episode, and 5-10\% of all women will experience recurrent vulvovaginal candidiasis ( $\geq 4$ episodes/year) (Rathod et al., 2012). In recent years there has been a tendency towards an increase of the number of relapses of this disease, in connection with which the analysis of causes, the development of diagnostic methods and the search for effective therapies for vaginal candidiasis has not lost its relevance (Diba et al., 2012; Sobel, 2016).

Candidiases are opportunistic mycoses, that is, they are caused by opportunistic fungi (Vijaya et al., 2014; Bradford \& Ravel, 2017). Of all the VCC cases, $80-90 \%$ are caused by $C$. albicans, and a minority are caused by C. glabrata, C. parapsilosis, and C. tropicalis (Qin et al., 2018). According to current notions, the indicated disease does not belong to the group of sexually transmitted diseases, but nevertheless the peak of incidence occurs at the beginning of sexual activity and the years of the greatest sexual activity of women. But the occurrence of this pathology often does not depend on the number of sexual partners and the frequency of sexual contacts (Hedayati et al., 2015). The morbidity associated with vulvovaginal candidiasis is not a cause of mortality, but makes it a major cause of mental distress and economic costs (Rathod \& Buffler, 2014). Vulvovaginal candidiasis, as a disease caused by opportunistic pathogens, usually occurs when the human immune mechanisms are affected by disorders, for example, it occurs in women during pregnancy. Thus, during pregnancy due to changes in hormonal balance and immunosuppression effect of progesterone, the incidence of vaginal candidiasis increases by 2-3 times (Bitew \& Abebaw, 2018). Among the factors that lead to the development of candidiasis, experts highlight: obesity, wearing tight clothing, non-compliance with the rules of personal hygiene, hot climate etc. (Richter et al., 2005).

Changing the permeability of the fungal cell membrane is the main effect of antifungal drugs. Nowadays mainly two groups of antifungal drugs are used to treat vulvovaginal candidiasis: polyene and pyrrole ring antifungal drugs. The first group is represented by amphotericin B, which has a wide antibacterial spectrum and a strong antifungal activity, but it is quite toxic. The second group are azoles (for example, ketoconazole, fluconazole, and itraconazole). These also have a wide spectrum. Now it is the most widely used group of antifungal drugs (Qin et al., 2018).

One more group of drugs are also being studied - echinocandins. Echinocandins are a new class of antifungal drugs that inhibit the synthesis of $\beta$-glucan in the fungal cell wall via noncompetitive inhibition 
of the enzyme 1,3- $\beta$ glucan synthase (Morris \& Villmann, 2006). They can influence a wide spectrum of Candida yeasts, and microorganisms of this genus are not broadly resistant to them. Therapeutic concentrations of representatives of group are smaller compared to other antifungal drugs (Perlin, 2014; Boikov et al., 2017). But at the same time, echinocandins have some significant disadvantages: they were found to be embryotoxic in animal studies (category C) thus should be avoided if possible in pregnancy, need dose adjustment in cases of liver disease and have poor ocular penetration in fungal endophthalmitis (Long, 2003).

Among the Candida yeasts, there is a rather high level of resistance to certain antimycotic drugs, in particular to fluconazole, which requires a preliminary study of the sensitivity of isolated strains to antimycotics for the development of the rational and effective treatment regimen (Deorukhkar et al., 2014; Liu et al., 2014; Monroy-Pérez et al., 2016; Rezaei-Matehkolaei et al., 2016; Zazharskyi et al., 2019).

In view of this, the objective of the research was studying the species range of fungi of the genus Candida isolated from the reproductive tract during dysbiosis and to study their susceptibility to antimycotics.

\section{Materials and methods}

The research was conducted in the laboratory of PE "Center of laboratory medicine "VIS-MEDIC" (Dnipro, Ukraine). The samples of biological material (secretion of vaginal mucosa) was obtained from women with pathological processes of the genital tract, the etiology of which had to be established. Materials were obtained in 2018 from women aged 16 to 56 years $(n=227)$. The specimens were taken in accordance to the standards of biomedical ethics.

The study of the material taken from the mucous membrane included microscopic analysis and determination of the Candida species by the polymerase chain reaction (PCR) method. For all isolated strains of fungi the definition of susceptibility to antimycotic drugs was also carried out (Clinical..., 2009). Preparations for microscopy were prepared by swirling of a tampon with material on the surface of an object glass. Three preparations were made:

- unstained - for seeing the presence of pseudomyces, chlamydospores and blastospores (microscopy by ${ }^{\times} 200$ );

- stained by Giemsa method: yeast-like fungal cells are pinkishpurple, chromatin is red, and volutin granules are dark violet. Separate cells (their shape, size, and status) were studied (microscopy by ${ }^{\times} 900$ );

- stained by Gram method - to assess the presence of other microorganisms for the diagnosis of dysbiosis according to the criteria of $\mathrm{Nu}-$ gent score. In smears stained by Gram method, the dark-purple or uneven colouration of cells of yeast morphology was determined (microscopy by ${ }^{\times}$900).

Positive results of microscopy are the detection of round and oval cells, many of which are found in the process of budding. Besides the cellular elements, the presence of pseudomycelium was determined (the chain of cells were not separated from each other), often with bloating due to budding. The location of yeast cells by clusters in the form of pockets along the pseudomycelium in the places where it joins is typical.

Detection of a large number of cells budding on the pseudomycelium is an important diagnostic symptom, indicating precisely the infectious process. When acute lesions on the vaginal mucosa take place, there are accumulation of large numbers of budded blastospores and pseudomycelium. The presence of single budding cells indicates candidiasis. Candidiasis was also determined in the absence of pseudomycelium, but by the presence of blastospores only (Mendling et al., 2015).

The identification of fungi was carried out using the RealBest DNA Candida albicans/Fungi and RealBest DNA Candida krusei/Candida glabrata test kits (JSC Vector-Best, Russia) by the methods of molecular diagnostics using a polymerase chain reaction (PCR) in real-time. This was done by iCycler iQ ${ }^{\mathrm{TM}} 5$ (Bio-Rad, USA) for performing PCR with real-time results detection in accordance with the manufacturer's instructions. Qualitative and quantitative indexes of the presence of Candida species in the samples were obtained. The criteria of candidiasis was the detection of fungi in more than $10^{3} \mathrm{CFU} / \mathrm{mL}$.

Material for identification was obtained by growing isolated colonies of fungi on Saburo medium (HiMedia, India). The surface of colo- nies (smooth, rough, wrinkled), pigment, consistency and the presence of chlamidospores and pseudomycelium were taken into account. The colonies which were later tested for the species $C$. albicans, on the Saburo medium had a colour from whitish-cream to snow-white, and were smooth and shiny. As non-albicans Candida species pre-determined rough colonies, this material was tested for belonging to the species C. krusei and C. glabrata. Cultures with a typical morphotype of Candida that did not give a positive response when determining the species by PCR were marked as Candida spp.

After identifying the isolates and determining their significance as an etiological factor of vaginal dysbiosis, the study of the susceptibility of the culture to the antimycotics was carried out. The susceptibility of the isolated strains was determined by a disk diffusion method (Method..., 2009) using standard disks with amphotericin B, ketoconazole, itraconazole, clotrimazole, nystatin and fluconazole (HiMedia, India).

\section{Results}

A total of 227 samples of biological material from women with reproductive tract pathology and complaints of itching, burning, purulent edema in the perineum were investigated. At the first stage, microscopy of the material was performed. According to the results of microscopic analysis, the presence of yeast-like fungi Candida in 174 samples of the material was determined, which was $76.7 \%$. In the study of microscopic dyes stained by the Gram method, the presence of other microorganisms was established, which prevents the identification of fungi as a monoculture, which determines the dysbiosis of the female vagina. The predominance of Candida presence was determined for 38 (21.8\%) samples of biological material.

Microscopy of non-stained preparations allowed visualization of the presence of pseudomycelium, chlamydospores and blastospores. The presence of these objects allowed us to pre-determine the infectious process in $76(43.7 \%)$ women. For other specimens, these signs were not marked, which made it possible to make a preliminary conclusion about carriage of Candida in 98 (56.3\%) cases. On the Gram-stained preparations, vaginal epithelium cells and concomitant microflora were present. In 115 (66.1\%) cases a large number of epithelial cells, surrounded by small gram negative microflora ("key cells") was noted, which allowed us to confirm dysbiosis. By the analysis of the obtained data it was established that the incidence of Candida carriage (56.3\%) exceeds the frequency of detection of active Candida infection (43.7\%). Such distribution is probably due to the fact that Candida infection, even in the absence of treatment, often occurs asymptomatically and women do not seek medical assistance, which leads to chronic infection.

Microscopic diagnostics in questionable and in doubtful cases should be confirmed by laboratory isolation of pure culture or by means of molecular genetic analysis. The real-time PCR method is used to establish species diversity. All the cultures grown on Saburo medium were identified as belonging to C. albicans, C. glabrata, C. krusei or Candida spp. (Fig. 1).

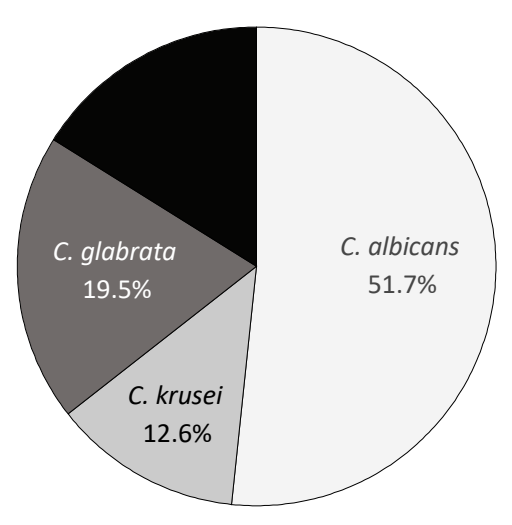

Fig. 1. Species composition of Candida fungi, isolated from vagina $(n=174)$ 
It was found that $C$. albicans was the predominant species, whose frequency of detection was more than half of cases - $90(51.7 \%)$. Other Candida species were C. glabrata - 34 (19.5\%) and C. krusei - 22 $(12.6 \%)$ cultures. 28 other cultures were identified as Candida spp. (16.1\%). The quantitative studies allowed us to confirm the infectious process (the number of fungal cells in the vaginal secretion exceeded $10^{3} \mathrm{CFU} / \mathrm{mL}$ ) in all cases of positive microscopic analysis, as well as in 8 cases, where microscopy revealled carriage of fungi. Consequently, the final frequency of Candida carriage was $51.7 \%$, and the frequency of infection was $48.3 \%$. In this case, C. albicans prevailed in infectious lesions, which were detected in excess of $10^{3} \mathrm{CFU} / \mathrm{mL}$ : 64 (76.2\%) cases (Fig. 2). Other Candida species were found predominantly in carriage - 58 (64.4\%) cases of 90 . In the infectious process, C. glabrata, C. krusei and Candida spp. were determined predominantly in the amount of $10^{3}$ $10^{6} \mathrm{CFU} / \mathrm{mL}$. Excess in the index of more than $10^{6} \mathrm{CFU} / \mathrm{mL}$ for these microorganisms was noted in exceptional cases.

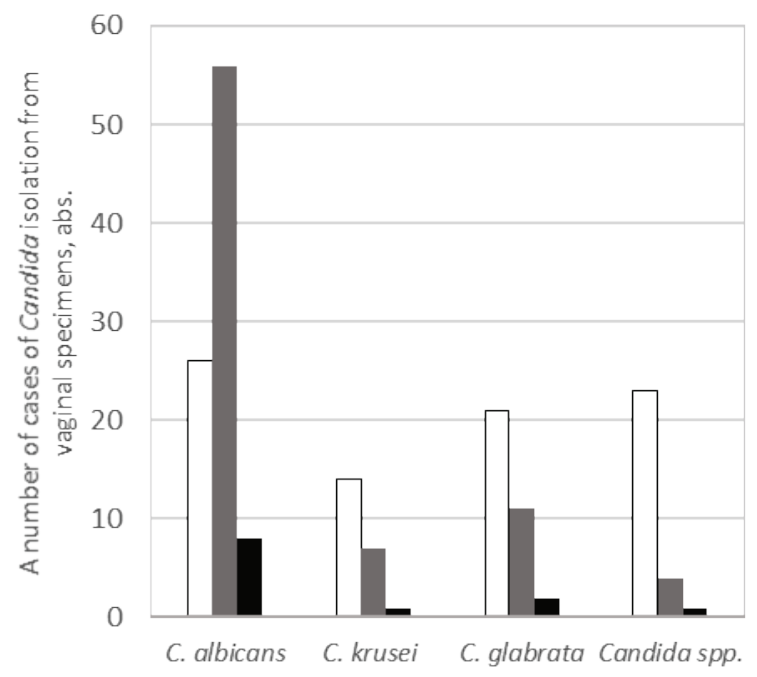

Fig. 2. The number of cases of isolation of various Candida species in the carriage and infectious process: white $-<10^{3} \mathrm{CFU} / \mathrm{mL}$; grey $-10^{3}-10^{6} \mathrm{CFU} / \mathrm{mL}$; black $->10^{6} \mathrm{CFU} / \mathrm{mL}$

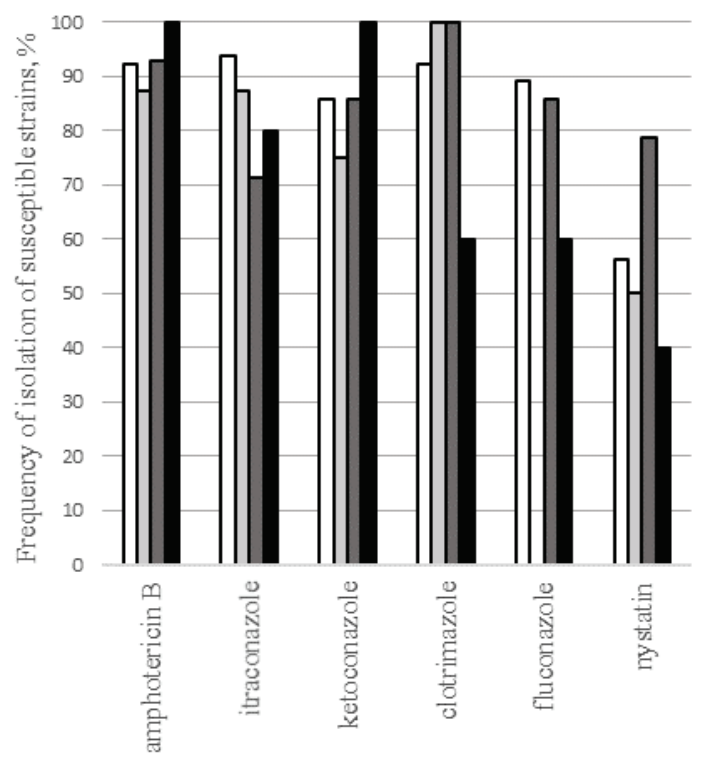

Fig. 3. Susceptibility to antimycotics of Candida strains, isolated from vagina of women in carriage and candidiasis: white - C. albicans; light grey - C. krusei;

dark grey - C. glabrata; black - Candida spp

For all Candida cultures, the amount of which in the material exceeded $10^{3} \mathrm{CFU} / \mathrm{mL}$, an antibiotic susceptibility study was performed, which is necessary for further development of the scheme of rational antibiotic therapy. The study of susceptibility to antimycotic drugs has made it possible to determine that resistance is not typical for the surveyed species (Fig. 3). The exception was $C$. krusei, which has natural resistance to fluconazole.

It is evident from data the presented in Figure 3 that the percentage of susceptible isolates with the exception of nystatin is quite large (more than $60-70 \%$ ) and is a good predictor for the treatment of vulvovaginal candidiasis. The exception is the only drug - nystatin, for which the number of susceptible strains of fungi does not reach $60 \%$. The least number of susceptible isolates were among Candida spp. $-40 \%$ and C. krusei- $-50 \%$. Consequently, the use of this drug for the treatment of women will not be effective and other options should be considered. All isolates were the most susceptible to amphotericin B: more than $87 \%$, but this antimycotic is not used in gynecology. For other strains, virtually all drugs have been effective, which allows a wide reserve among the drugs of choice. For $C$. krusei, the drug of choice is itraconazole, to which $98.5 \%$ of isolates were sensitive.

\section{Discussion}

Yeast-like fungi Candida are opportunistic microorganisms that cause diseases in carriers in the event of damage to their immune system (Sobel, 2016). Vulvovaginal candidiasis is important in obstetric and gynecological practice: more than $70 \%$ of women at least once in their life suffer from this illness (Hedayati et al., 2015). It is known that most common pathogens which provoke candidiasis are $C$. albicans, C. krusei and C. glabrata. But we found many other species that we described as Candida spp. It should be noted that in the latter group it is possible to detect a sufficiently large number of Candida species, in particular C. tropicalis, C. parapsilosis, C. famata, C. lusitaniae, C. kefyr, C. guilliermondii. However, in most cases, their presence does not exceed $3-5 \%$ in the general structure of pathogens causing vulvovaginal candidiasis (Fornari et al., 2012; Bitew \& Abebaw, 2018). Indeed, vulvovaginal candidiasis is predominantly caused by $C$. albicans, and other Candida species occur much less frequently. According to various data (Bradford \& Ravel, 2017; Bitew \& Abebaw, 2018), the detection rate of $C$. albicans is at least $35-45 \%$, which is confirmed by the results obtained by us. Accordingly, the total frequency of detection of nonalbicans Candida species is more than 50\% (Liu et al., 2014; Khan et al., 2018). Separately, species such as C. glabrata and C. krusei are quite common. The frequency of their detection is $10-20 \%$ (Hedayati et al., 2015; Wang et al., 2016; Bradford \& Ravel, 2017). Most other species are often combined in Candida spp., whose share is formed by species, the frequency of which does not exceed 2-3\% (Gamarra et al., 2014; Wang et al., 2016).

The disease occurs in women of all age groups, often during pregnancy. However, the urgency of the problem is due not only to its significant prevalence (Corsello et al., 2003). Vulvovaginal candidiasis is often combined with other disorders of the microbiocenosis of the vagina and is associated with the threat of abortion, involuntary miscarriage, premature delivery, chorioamnionitis, untimely discharge of amniotic fluid, the birth of children with low body weight, chronic hypoxia or signs of intrauterine infection, the onset of wound infection of the birth canal, endometritis in the postpartum period. Healthy pregnant women and pregnant women with vaginal vaginal candidiasis can be a source of both intrauterine and postnatal infections in newborns (Bitew \& Abebaw, 2018).

The use of antifungal drugs is a mandatory step in the treatment of vulvovaginal candidiasis. Under conditions of successful prescription of drugs it is possible to remove yeast from the vaginal biotope. However, in case of non-compliance with the prescribed course of treatment or in case of unsuccessful use of the drug, carriage is formed, which is much more difficult to get rid of than active infection. The best therapy to use is local, but the success of therapy will be guaranteed only if the sensitivity of the selected culture to the available antifungal drugs is determined (Gamarra et al., 2014; Fornari et al., 2016). Nowadays there is a tendency towards the spread of candidiasis, and therefore the problem of its effective treatment needs special attention. Taking into account the spread of resistance to medicines among the Candida strains, the treat- 
ment of candidiasis is becoming a difficult task, which cannot be based solely on canonical ideas about the mechanism of action of drugs (Deorukhkar et al., 2014; Liu et al., 2014). There is a need to study the level of sensitivity to the antimycotics of each particular isolate (Abbasi Nejat et al., 2018). In addition, attention should be paid to the treatment of concomitant diseases that can complicate the course of candidiasis. One of the main conditions for the treatment period is, if possible, the avoidance of corticosteroids, cytostatics, hormonal estrogen-progestin preparations, antibiotics, and the abandonment of bad habits. It is necessary to perform correction of the conditions that lead to the development of genital candidiasis. Use of multivitamin complexes may be beneficial (Mendling et al., 2015; Pappas et al., 2016; Khan et al., 2018).

Therapy of vulvovaginal candidiasis should be comprehensive and phased. The advantage of local medications is that they are practically not absorbed, create a high concentration of antimycotics on the mucous membrane, provide a rapid reduction of clinical symptoms. Therefore, their use is justified in pregnancy and lactation. In modern clinical practice, for the treatment of vulvovaginal candidiasis, fluconazole and ketoconazole are used, and somewhat less clotrimazole is used for the treatment of fungal skin lesions (Mendling et al., 2015; Pappas et al., 2016), therefore these preparations were a compulsory choice for research. The disadvantages of local therapy include uneven distribution of the drug over the surface of the mucous membrane, which creates conditions for the preservation of the reservoir of infection. In the application of topical antimycotics, systemic elimination of candidiasis infection in other reservoirs is not provided, which may lead to a recurrence of infection (Richter et al., 2005; Diba et al., 2012; Sobel, 2016). Many patients point to the inconvenience of using local therapy, which reduces the quality of life. The listed shortcomings of local means determine the relevance of systemic therapy (Richter et al., 2005; Vijaya et al., 2014).

The advantage of systemic antimycotics is the high effectiveness of treatment, distribution in many organs and tissues, and hence the effect on the pathogen in any localization. One of the important advantages of systemic antimycotics is the convenience of use in comparison with local vaginal forms (Mendling et al., 2015; Pappas et al., 2016; RezaeiMatehkolaei et al., 2016). Given the increased resistance of Candida to antimycotics, in our time, systemic therapy for candidiasis should be based on the susceptibility of the pathogen (Rathod et al., 2012). For example, C. krusei is genetically resistant to fluconazole (Güzel et al., 2013), and C. glabrata is dose-dependent to fluconazole, and only when it is used in a dose of $400-800 \mathrm{mg}$ per day is it possible to eliminate the pathogen. In this way, if it is not possible to conduct a cultural diagnosis, the treatment should be carried out using antimycotics of the widest possible range of action (Pappas et al., 2016).

\section{Conclusions}

The frequency of detection of Candida species in the composition of microbiota of the reproductive tract of women was $76.7 \%$ in patients with clinical manifestation of vaginal discharges. The incidence of Candida carriage $(51.7 \%)$ was slightly prevalent over the incidence of active infection (48.3\%). The most common pathogen was $C$. albicans, which was found in $57.1 \%$ of cases, which corresponds to the typical spread of this pathogen among women of reproductive age in different countries. Also, C. glabrata (19.5\%), C. krusei (12.6\%) and Candida spp. (16.1\%) were the cause of pathological processes. In active infectious processes, C. albicans (64/76.2\% cases) predominated. Other species of Candida were most commonly detected in carriage $(58 /$ $64.4 \%$ cases), while they can be typical for an active infection too. All isolates showed the maximal susceptibility to amphotericin B and the least susceptibility to nystatin.

\section{References}

Abbasi Nejat, Z., Farahyar, S., Falahati, M., Ashrafi Khozani, M., Hosseini, A. F., Faiazy, A., Ekhtiari, M., \& Hashemi-Hafshenjani, S. (2018). Molecular identification and antifungal susceptibility pattern of non-albicans Candida species isolated from vulvovaginal candidiasis. Iranian Biomedical Journal, 22(1), 33-41.
Alfouzan, W., Dhar, R., Ashkanani, H., Gupta, M., Rachel, C., \& Khan, Z. U. (2015). Species spectrum and antifungal susceptibility profile of vaginal isolates of Candida in Kuwditurnal de Mycologie Médiçale, 23-28.

Bitew, A., \& Abebaw, Y. (2018). Vulvovaginal candidiasis: Species distribution of Candida and their antifungal susceptibility pattern. BMC Women's Health, 18(1), 94.

Boikov, D. A., Locke, J. B., James, K. D., Bartizal, K., \& Sobel, J. D. (2017). In vitro activity of the novel echinocandin CD101 at $\mathrm{pH} 7$ and 4 against Candi$d a$ spp. isolates from patients with vulvovaginal candidiasis. The Journal of Antimicrobial Chemotherapy, 72(5), 1355-1358.

Bradford, L. L., \& Ravel, J. (2017). The vaginal mycobiome: A contemporary perspective on fungi in women's health and diseases. Virulence, 8(3), 342-351.

Corsello, S., Spinillo, A., Osnengo, G., Penna, C., Guaschino, S., Beltrame, A., Blasi, N., \& Festa, A. (2003). An epidemiological survey of vulvovaginal candidiasis in Italy. European Journal of Obstetrics, Gynecology, and Reproductive Biology, 110(1), 66-72.

Deorukhkar, S. C., Saini, S., \& Mathew, S. (2014). Non-albicans Candida infection: An emerging threat. Interdisciplinary Perspectives on Infectious Diseases, 2014, 615958 .

Diba, K., Namaki, A., Ayatolahi, H., \& Hanifian, H. (2012). Rapid identification of drug resistant Candida species causing recurrent vulvovaginal candidiasis. Medical Mycology Journal, 53, 193-198.

Fornari, G., Vicente, V. A., Gomes, R. R., Muro, M. D., Pinheiro, R. L., Ferrari, C., Herkert, P. F., Takimura, M., Carvalho, N. S., \& Queiroz-Telles, F. (2016). Susceptibility and molecular characterization of Candida species from patients with vulvovaginitis. Brazilian Journal of Microbiology, 47(2), 373-380.

Gamarra, S., Morano, S., Dudiuk, C., Mancilla, E., Nardin, M. E., de Los Angeles Mendez, E., \& Garcia-Effron, G. (2014). Epidemiology and antifungal susceptibilities of yeasts causing vulvovaginitis in a teaching hospital. Mycopathologia, 178(3-4), 251-258

Güzel, A. B., Aydın, M., Meral, M., Kalkancı, A., \& Ilkit, M. (2013). Clinical characteristics of turkish women with Candida krusei vaginitis and antifungal susceptibility of the $C$. krusei isolates. Infectious Diseases in Obstetrics and Gynecology, 2013, 698736.

Hedayati, M. T., Taheri, Z., Galinimoghadam, T., Aghili, S. R., Cherati, J. Y., \& Mosayebi1, E. (2015). Isolation of different species of Candida in patients with vulvovaginal candidiasis from Sari, Iran. Jundishapur Journal of Microbiology, 8(4), e15992.

Khan, M., Ahmed, J., Gul, A., Ikram, A., \& Lalani, F. K. (2018). Antifungal susceptibility testing of vulvovaginal Candida species among women attending antenatal clinic in tertiary care hospitals of Peshawar. Infection and Drug Resistance, 11, 447-456.

Liu, X. P., Fan, S. R., Peng, Y. T., \& Zhang, H. P. (2014). Species distribution and susceptibility of Candida isolates from patient with vulvovaginal candidiasis in Southern China from2003 to 2012 Journal de Mycologie Médicą24, $106-111$.

Long, J. (2003). Echinocandins. In: Wyman, M. J., Leonard, M. C., \& Lehmann, M. K. (Eds.). New antifungal agents additions to the existing armamentarium (Part 1). Center for Continuing Education, Lyndhurst.

Mendling, W., Friese, K., Mylonas, I., Weissenbacher, E. R., Brasch, J., Schaller, M., Mayser, P., Effendy, I., Ginter-Hanselmayer, G., Hof, H., Cornely, O., \& Ruhnke, M. (2015). Vulvovaginal candidosis (excluding chronic mucocutaneous candidosis) guideline of the German Society of Gynecology and Obstetrics (AWMF Registry No 015/072, S2k Level, December 2013) Geburtshilfe Frauenheilkd. 75(4), 342-354

Method for antifungal disk diffusion susceptibility testing of yeasts; approved guideline (CLSI document M44-A2). (2009). CLSI, Wayne.

Monroy-Pérez, E., Luz Paniagua-Contreras, G., Rodríguez-Purata, P., VacaPaniagua, F., Vázquez-Villaseñor, M., Díaz-Velásquez, C., Uribe-García, A., \& Vaca, S. (2016). High virulence and antifungal resistance in clinical strains of Candida albicans. Canadian Journal of Infectious Diseases and Medical Microbiology, 2016, 5930489.

Morris, M. I., \& Villmann, M. (2006). Echinocandins in the management of invasive fungal infections, Part 2. American Journal of Health-System Pharmacy, 63(19), 1813-1820.

Pappas, P. G., Kauffman, C. A., Andes, D. R., Clancy, C. J., Marr, K. A., OstroskyZeichner, L., Reboli, A. C., Schuster, M. G., Vazquez, J. A., Walsh, T. J., Zaoutis, T. E., \& Sobel, J. D. (2016). Clinical practice guideline for the management of candidiasis: 2016 Update by the Infectious Diseases Society of America. Clinical Infectious Diseases, 62(4), e1-e50.

Perlin, D. S. (2014). Echinocandin resistance, susceptibility testing and prophylaxis: Implications for patient management. Drugs, 74(14), 1573-1585.

Qin, F., Wang, Q., Zhang, C., Fang, C., Zhang, L., Chen, H., Zhang, M., \& Cheng, F. (2018). Efficacy of antifungal drugs in the treatment of vulvovaginal candidiasis: a Bayesian network meta-analysis. Infection and Drug Resistance, 11, 1893-1901. 
Rathod, S. D., \& Buffler, P. A. (2014). Highly-cited estimates of the cumulative incidence and recurrence of vulvovaginal candidiasis are inadequately documented. BMC Women's Health, 14(1), 43.

Rathod, S. D., Klausner, J. D., Krupp, K., Reingold, A. L., \& Madhivanan, P. (2012) Epidemiologic features of vulvovaginal candidiasis among reproductive-age women in India. Infectious Diseases in Obstetrics and Gynecology, 2012, 859071.

Rezaei-Matehkolaei, A., Shafiei, S., \& Zarei-Mahmoudabadi, A. (2016). Isolation, molecular identification, and antifungal susceptibility profiles of vaginal isolates of Candida species. Iranian Journal of Microbiology, 8(6), 410-417.

Richter, S. S., Galask, R. P., Messer, S. A., Hollis, R. J., Diekema, D. J., \& Pfaller, M. A. (2005). Antifungal susceptibilities of Candida species causing vulvo- vaginitis and epidemiology of recurrent cases. Journal of Clinical Microbiology, 43, 2155-2162

Sobel, J. D. (2016). Recurrent vulvovaginal candidiasis. American Journal of Obstetrics and Gynecology, 214, 15-21.

Vijaya, D., Dhanalakshmi, T. A., \& Kulkarni, S. (2014). Changing trends of vulvovaginal candidiasis. Journal of Laboratory Physicians, 6(1), 28-30.

Wang, F. J., Zhang, D., Liu, Z. H., Wu, W. X., Bai, H. H., \& Dong, H. Y. (2016) Species distribution and in vitro antifungal susceptibility of vulvovaginal Candida isolates in China. Chinese Medical Journal, 129(10), 1161-1165.

Zazharskyi, V. V., Davydenko, P. O., Kulishenko, O. M., Borovik, I. V., \& Brygadyrenko, V. V. (2019). Antimicrobial activity of 50 plant extracts. Biosystems Diversity, 27(2), 163-169. 